\title{
Comparación Clínica entre el Tratamiento Ortodóncico Facilitado por Corticotomía y Ortodoncia Convencional (Estudio Piloto)
}

\author{
Clinical Comparison of Orthodontic Treatment Facilitated by \\ Corticotomy and Conventional Orthodontics (Pilot Study)
}

\begin{abstract}
Juan David Arango*; Claudia María Roldan*; Luz María Burgos*; Carolina Giraldo*; Carlos Efraín Gutiérrez*; Luis Alejandro Sánchez*; Mauricio Villegas;; David Arango*; Maryori Restrepo* \& Javier Enrique Botero*
\end{abstract}

\begin{abstract}
ARANGO, J. D.; ROLDAN, C. M.; BURGOS, L. M.; GIRALDO, C.; GUTIÉRREZ, C. E.; SÁNCHEZ, L. A.; VILLEGAS, M.; ARANGO, D.; RESTREPO, M. \& BOTERO, J. E. Comparación clínica entre el tratamiento ortodóncico facilitado por corticotomía y ortodoncia convencional (estudio piloto). Int. J. Odontostomat., 9(2):239-248, 2015.

RESUMEN: El objetivo de este estudio fue comparar clínicamente la velocidad del movimiento ortodóncico y cambios en los parámetros periodontales en pacientes con ortodoncia convencional y ortodoncia facilitada con corticotomía para el tratamiento de apiñamiento dental anterior. Un total de 10 pacientes participaron en el estudio de la siguiente forma: 5 pacientes grupo ortodoncia y corticotomía (EXP) y 5 pacientes grupo ortodoncia (CONT). Se realizó examen clínico completo que incluyó análisis radiográfico, periodontal y modelos de estudio. Las mediciones sobre los cambios periodontal, tabla ósea bucal, movimiento lineal y angular fueron medidos al inicio, 30, 90 y 210 días posterior a los procedimientos. Las diferencias entre los grupos fueron establecidas con la prueba t-Student para muestras pareadas y no pareadas dependiendo del caso. Los dientes sometidos a ortodoncia y corticotomía mostraron una mayor velocidad del movimiento en comparación con el grupo control durante los primero 30 días de examinación $(P<0,05)$. No hubo diferencias estadísticamente significativas en los parámetros clínicos periodontales y grosor de la tabla ósea bucal entre los grupos. En conclusión, la corticotomía acelera el movimiento ortodóncico durante los primero 30 días postquirúrgicos con lo cual se puede reducir el tiempo de tratamiento en pacientes con apiñamiento severo. Adicionalmente, los parámetros clínicos periodontales y el volumen de la tabla ósea bucal de los dientes sometidos a corticotomía se mantienen estables después del procedimiento.
\end{abstract}

PALABRAS CLAVE: corticotomía, ortodoncia acelerada, parámetros clínicos periodontales, fenómeno acelerado regional (RAP).

\section{INTRODUCCIÓN}

El apiñamiento dental es una condición caracterizada por la falta de espacio para la correcta alineación de los dientes, situación que presenta alta prevalencia en el grupo poblacional de los 12 y 19 (Siluvai et al., 2014; Hanna et al., 2015). Esta alteración se comienza a desarrollar temprano en los jóvenes que generalmente llegan a la edad adulta sin recibir tratamiento, lo cual favorece la acumulación de placa bacteriana y desarrollo de enfermedades periodontales (Dhar et al., 2007). Adicionalmente, el apiñamiento dental es responsable de una alteración en la estética de los pacientes, lo cual puede generar problemas de autoestima (Jung et al., 2015).
La corrección del apiñamiento dental se lleva a cabo por medio del tratamiento ortodóncico. No obstante, el tiempo de duración de los tratamientos es una preocupación de los pacientes y más aun en los pacientes adultos, pues el tratamiento puede ser complejo (Christensen \& Luther, 2015). Lo anterior debido a perdida de dientes, perdida de soporte y adaptabilidad fisiológica propia de los tejidos periodontales jóvenes, lo cual aumenta en el tiempo de tratamiento ortodóncico (Panwar et al., 2014). Es claro entonces, que siendo las maloclusiones en adultos una causa frecuente de consulta en ortodoncia (Buttke \& Proffit, 1999), disminuir el tiempo de tratamiento es deseable, ya que al hacerlo,

* Facultad de Odontología, Universidad de Antioquia, Medellín, Colombia. 
ARANGO, J. D.; ROLDAN, C. M.; BURGOS, L. M.; GIRALDO, C.; GUTIÉRREZ, C. E.; SÁNCHEZ, L. A.; VILLEGAS, M.; ARANGO, D.; RESTREPO, M. \& BOTERO, J. E. Comparación clínica entre el tratamiento ortodóncico facilitado por corticotomía y ortodoncia convencional (estudio piloto). Int. J. Odontostomat., 9(2):239-248, 2015.

se disminuye el riesgo de caries, inflamación gingival y reabsorción radicular (Consolaro, 2013; Panwar et al.).

Uno de los métodos reportados para reducir la duración del tratamiento es la ortodoncia asistida por corticotomía (OAC) (Buschang et al., 2012). La corticotomía se define como el procedimiento quirúrgico de osteotomía controlada y limitada a la tabla ósea cortical, generalmente la bucal, poco invasiva, que como ventaja adicional permite un patrón de remodelado óseo con poco riesgo de daño a los tejidos periodontales (Gantes et al., 1990; Bushang et al.). El propósito de la corticotomía en combinación con fuerzas ortodóncicas es acelerar el movimiento dental (Bushang et al.). La explicación del aumento en el movimiento ortodóncico se encuentra en un proceso denominado Fenómeno de Aceleración Regional (FAR) (Wang et al., 2009). Este concepto propone que el hueso decorticado se desmineraliza y presenta una fase de remodelación (34 meses) como respuesta del tejido ante el trauma quirúrgico inducido lo cual reduce la resistencia a las fuerzas ortodoncias, tiempo que se aprovecha para el movimiento rápido de los dientes.

Las indicaciones clínicas para el uso de la OAC han sido amplias, reportando éxito en el manejo de expansión palatina asistida quirúrgicamente, protrusión bimaxilar dentoalveolar, mordida abierta anterior, protrusión esquelética severa y apiñamiento dental, adicionalmente se usa en pacientes adultos para acelerar el tiempo de tratamiento ortodóncico (Owen, 2001; Wilcko et al., 2001; Byloff \& Mossaz, 2004; Lee et al., 2007; Akay et al., 2009).

Sin embargo, los estudios realizados hasta ahora en humanos son escasos, sin estandarización de la técnica y con poco tiempo de seguimiento de resultados (Nowzari et al., 2008; Chung et al., 2009). Esto sugiere una falta de evidencia científica para que este procedimiento pueda ser aplicado de manera confiable a los tratamientos de ortodoncia. El objetivo de este estudio fue comparar clínicamente de la velocidad del movimiento ortodóncico y cambios en los parámetros periodontales en pacientes con ortodoncia convencional y ortodoncia facilitada con corticotomía (OAC) para el tratamiento de apiñamiento dental anterior.

\section{MATERIAL Y MÉTODO}

Se diseñó un estudio clínico piloto con una muestra por conveniencia de 10 sujetos, 5 en el grupo ortodoncia + corticotomía (EXP) y 5 en el grupo ortodoncia (CONT). Debido a la naturaleza de la intervención quirúrgica, no fue posible hacer aleatorización, y solo se realizó el procedimiento en sujetos que aceptaran voluntariamente el procedimiento. No obstante, el estudio está registrado en Clinical Trials por tratarse de un estudio de intervención en humanos (NCT01630473). Así mismo, el protocolo de investigación fue aprobado por el comité de ética de la Facultad de Odontología de la Universidad de Antioquia. Para la inclusión en el estudio los pacientes firmaron un consentimiento escrito informado y siguiendo las recomendaciones de la declaración de Helsinki (2009) para estudios en humanos.

Para el reclutamiento de pacientes, se invitó a participar sujetos evaluados en las clínicas odontológicas de la Facultad de Odontología de la Universidad de Antioquía entre enero de 2012 y diciembre de 2013. Durante la clasificación de posibles voluntarios se siguieron los siguientes criterios de inclusión: paciente hombre o mujer de 20 a 40 años, con dentición permanente completa, con apiñamiento de leve a severo, con biotipo periodontal grueso o mixto, y que aceptaran de forma voluntaria el procedimiento quirúrgico como parte del tratamiento. Los voluntarios fueron excluidos cuando presentaran las siguientes características: enfermedad sistémica o antecedentes médicos que contraindiquen la cirugía; consumo de bisfosfonatos, antiepilépticos, benzodiacepinas, inhibidores o inductores enzimáticos, glucocorticoides, estrógenos, anti-estrógenos, anticonceptivos, vitamina $\mathrm{D}$, calcitonina, sales de calcio, suplementos de calcio, corticoides, antihistamínicos, esteroides, con ortodoncia previa, con periodontitis, con pérdida de nivel de inserción periodontal y pérdida ósea de moderada a severa, con reabsorción radicular, pacientes fumadores y mujeres embarazadas.

Examen clínico periodontal. En ambos grupos se llevó a cabo un examen periodontal completo limitado a la zona anterio-superior y antero-inferior con una sonda periodontal UNC-15 (Delta, USA Chicago), registrando los siguientes parámetros clínicos:

1. Profundidad al sondaje (PD): medida en milímetros $(\mathrm{mm})$ desde el margen gingival hasta el fondo del surco.

2. Sangrado al sondaje (BOP): se calculó como el porcentaje (\%) de sitios que presenten sangrado positivo.

3. Margen gingival (MG): medida en $\mathrm{mm}$, como la distancia desde el margen hasta la línea amelocementaria. 
ARANGO, J. D.; ROLDAN, C. M.; BURGOS, L. M.; GIRALDO, C.; GUTIÉRREZ, C. E.; SÁNCHEZ, L. A.; VILLEGAS, M.; ARANGO, D.; RESTREPO, M. \& BOTERO, J. E. Comparación clínica entre el tratamiento ortodóncico facilitado por corticotomía y ortodoncia convencional (estudio piloto). Int. J. Odontostomat., 9(2):239-248, 2015.

4. Nivel de inserción (CAL): medida en mm, distancia de la unión amelocementaria hasta el fondo de surco.

5. Índice de placa (PI): después de aplicar pastilla reveladora, se calcula el porcentaje (\%) de sitios con placa.

6. Encía queratinizada (EQ): en $\mathrm{mm}$, desde el margen gingival hasta la unión mucogingival delimitada por la solución de Schiller.

La examinación periodontal fue realizada por un solo examinador (CEG) estandarizado y calibrado (coeficiente de correlación intraclase $>0,80$ ) en los tiempos T0 (inicio), T1 (30 días), T2 (90 días) y T3 (210 días) después de los procedimientos de quirúrgicos y de activación dependiendo del grupo.

Análisis radiográfico. Ambos grupos cumplieron el protocolo de ayudas diagnósticas que incluía radiografías (serie periapical, cefálica con trazado y panorámica), modelos de yeso digitalizados con el software Ortho Insight, fotos extra e intraorales y tomografía ConeBeam.

La evaluación tomográfica fue realizada en un equipo de Tomografía ConeBeam de marca NewTom 3G de fabricación Italiana, los parámetros de exposición utilizados para esta investigación fueron: Kv: 110; mA: 7,1; Dosis (mGy): 8,87; Detector: 9" (nueve pulgadas); Tamaño del voxel: $0,3 \mathrm{~mm}$; Escala de grises: 12 bit. Esta evaluación se hizo antes de realizar los procedimientos y 7 meses después de comenzado el tratamiento en ambos grupos. Las mediciones se realizaron por el evaluador estandarizado (CEG) bajo el protocolo descrito por Januário et al.. Las dimensiones de la pared ósea bucal fueron evaluadas en tres posiciones diferentes en relación con la cresta ósea así: a 1, 3, y $5 \mathrm{~mm}$ apical a la cresta ósea en el punto medio de cada diente anterior en el maxilar y mandíbula. Estas mediciones se realizaron utilizando el programa NNT Viewer, que es una versión ligera del programa NNT WorkStation el cual por medio de sus herramientas, permite la medición en $\mathrm{mm}$ del espesor de la tabla cortical bucal.

Tratamiento ortodóncico. Se realizó historia clínica completa con la definición del plan de tratamiento según las necesidades de cada paciente y se adhirió aparatología fija maxilomandibular, en todos los casos se utilizaron brackets slot 0,018 x 0,025 prescripción Roth 1 . El día 0 para el grupo control fue el día de adhesión de aparatología, mientras que para el grupo experimental el día 0 , fue el día de la cirugía en donde inmediatamente después se activa la ortodoncia con el primer arco.

Dentro del protocolo ortodóncico se definió una secuencia de arcos (Tabla I), que se adaptó a la evolución de cada paciente, teniendo en cuenta que el grupo experimental, siguió un cronograma de activación cada 15 días durante los primeros cuatro meses en relación con el aprovechamiento del RAP y el grupo control cada 30 días según los principios biológicos de la ortodoncia convencional.

A cada uno de los pacientes se realizó modelos de yeso, los cuales fueron escaneados y digitalizados con el programa Ortholnsight, Los movimientos dentarios en ambos grupos fueron medidos con cambios lineales y angulares. Para determinar los primeros, se relacionaron los valores lineales promedio en milímetros en los dientes 13-12-11-21-22-23, 33-32-31-41$42-43$,al centro de masa del modelo definido por el software. Adicionalmente se registraron las medidas angulares de los mismos dientes con vértice en el mismo centro de masa. Se registraron los datos tomados en 4 momentos: al inicio (T0) y 30 (T1), 90 (T2) y 210 (T3) después de los procedimientos quirúrgicos y de activación dependiendo del grupo.

El índice de irregularidad de Little (1975) fue determinado directamente en los modelos de yeso en los tiempos T1, T2 y T3 en ambos arcos.

Corticotomía. En el grupo EXP se realizó el procedimiento quirúrgico, 8 días después de adherida la aparatología, la cual se encontraba sin activar. Se realizó anestesia en el área anterior superior e inferior, por bucal y lingual/palatino con lidocaína más epinefrina al $2 \%$ y luego infiltración con dexametasona 1 ampolla de $4 \mathrm{mg}$ en la zona intervenida. A continuación, se realizó incisión intrasulcular con hoja de bisturí \# 15c (Hu-friedy) desde mesial de primeros molares (superiores e inferiores) hasta primeros molares contralaterales; consecutivamente se realizaron incisiones relajantes en mesial de primeros molares respetando íntegramente la papila y se levantó un colgajo de espesor completo bucal utilizando una micro legra "P Buser" (Delta, USA Chicago.) permitiendo acceder hasta la zona más apical de los dientes (mínimo $8 \mathrm{~mm}$ ). Posterior a esto, con una fresa 702 de carburo tallo largo en una pieza de baja velocidad bajo irrigación con jeringa desechable de $50 \mathrm{cc}$, se inició la perforación de la cortical ósea bucal en interproximal desde el 15 hasta el 25 y de 35 a 45, teniendo como precau- 
ARANGO, J. D.; ROLDAN, C. M.; BURGOS, L. M.; GIRALDO, C.; GUTIÉRREZ, C. E.; SÁNCHEZ, L. A.; VILLEGAS, M.; ARANGO, D.; RESTREPO, M. \& BOTERO, J. E. Comparación clínica entre el tratamiento ortodóncico facilitado por corticotomía y ortodoncia convencional (estudio piloto). Int. J. Odontostomat., 9(2):239-248, 2015.

Tabla I. Movimiento lineal en milímetros $(\mathrm{mm})$ por diente en el grupo corticotomía y control a los 210 días de observación.

\begin{tabular}{|c|c|c|c|c|c|c|}
\hline \multirow{2}{*}{ Diente } & \multicolumn{3}{|c|}{ Grupo corticotomía (Promedio \pm DE) } & \multicolumn{3}{|c|}{ Grupo control (Promedio \pm DE) } \\
\hline & T1 & T2 & T3 & T1 & T2 & T3 \\
\hline $13 \mathrm{M}$ & $3,74 \pm 2,39^{*}$ & $2,22 \pm 3,44$ & $4,62 \pm 2,09^{*}$ & $0,80 \pm 0,48$ & $1,28 \pm 0,80$ & $1,74 \pm 1,11$ \\
\hline $13 \mathrm{D}$ & $3,35 \pm 2,41$ & $1,61 \pm 2,38$ & $3,74 \pm 2,12$ & $0,55 \pm 0,72$ & $1,60 \pm 1,15$ & $1,45 \pm 1,04$ \\
\hline $12 \mathrm{M}$ & $4,44 \pm 2,16^{*}$ & $0,72 \pm 0,70$ & $4,11 \pm 2,29$ & $1,46 \pm 0,83$ & $1,88 \pm 1,39$ & $2,36 \pm 2,21$ \\
\hline $12 \mathrm{D}$ & $4,22 \pm 2,14^{*}$ & $0,96 \pm 1,08$ & $4,37 \pm 1,45$ & $1,19 \pm 0,64$ & $1,67 \pm 1,44$ & $2,39 \pm 1,34$ \\
\hline $11 \mathrm{M}$ & $4,13 \pm 0,60^{*}$ & $1,49 \pm 0,67$ & $3,46 \pm 1,74$ & $1,25 \pm 1,00$ & $1,42 \pm 1,36$ & $2,56 \pm 2,40$ \\
\hline 11D & $4,40 \pm 2,34^{*}$ & $1,45 \pm 0,93$ & $3,54 \pm 2,28$ & $1,70 \pm 0,94$ & $1,62 \pm 1,30$ & $2,93 \pm 2,25$ \\
\hline $21 \mathrm{M}$ & $3,40 \pm 1,24^{*}$ & $1,08 \pm 1,15$ & $3,57 \pm 1,95$ & $0,93 \pm 0,79$ & $2,09 \pm 1,63$ & $2,27 \pm 1,91$ \\
\hline 21D & $3,86 \pm 1,89$ & $1,42 \pm 0,50$ & $3,99 \pm 1,44$ & $1,55 \pm 1,39$ & $1,02 \pm 1,31$ & $2,09 \pm 1,57$ \\
\hline $22 \mathrm{M}$ & $4,34 \pm 2,58$ & $1,16 \pm 0,75$ & $3,89 \pm 2,60$ & $1,42 \pm 1,25$ & $0,82 \pm 1,15$ & $2,24 \pm 2,22$ \\
\hline $22 \mathrm{D}$ & $3,59 \pm 1,75^{\star}$ & $0,76 \pm 0,75$ & $3,10 \pm 1,79$ & $1,25 \pm 0,54$ & $0,72 \pm 1,06$ & $1,72 \pm 1,30$ \\
\hline $23 \mathrm{M}$ & $3,35 \pm 1,82^{*}$ & $0,82 \pm 0,39$ & $3,59 \pm 1,72^{*}$ & $1,27 \pm 0,60$ & $0,79 \pm 0,93$ & $1,13 \pm 0,50$ \\
\hline 23D & $2,74 \pm 1,59$ & $1,13 \pm 0,63$ & $3,39 \pm 1,89$ & $1,09 \pm 1,05$ & $0,88 \pm 0,46$ & $1,44 \pm 1,05$ \\
\hline Maxilar & $3,56 \pm 1,90^{*}$ & $1,23 \pm 1,11$ & $3,78 \pm 1,43$ & $1,20 \pm 0,85$ & $1,31 \pm 1,16$ & $2,02 \pm 1,53$ \\
\hline $33 \mathrm{M}$ & $3,51 \pm 1,48$ & $0,75 \pm 0,73$ & $3,52 \pm 1,63$ & $1,72 \pm 1,11$ & $1,78 \pm 1,37$ & $2,54 \pm 1,88$ \\
\hline 33D & $2,83 \pm 1,80$ & $0,92 \pm 0,92$ & $2,80 \pm 1,75$ & $1,20 \pm 0,64$ & $1,17 \pm 0,39$ & $1,31 \pm 1,45$ \\
\hline $32 \mathrm{M}$ & $3,94 \pm 1,10^{*}$ & $0,84 \pm 0,71$ & $3,46 \pm 1,72$ & $1,77 \pm 1,32$ & $2,26 \pm 2,06$ & $3,06 \pm 3,38$ \\
\hline $32 \mathrm{D}$ & $3,84 \pm 1,47^{*}$ & $1,37 \pm 0,81$ & $4,12 \pm 2,95$ & $1,52 \pm 1,02$ & $1,55 \pm 1,66$ & $2,79 \pm 1,92$ \\
\hline $31 \mathrm{M}$ & $4,27 \pm 2,58^{*}$ & $0,97 \pm 0,63$ & $4,10 \pm 2,05$ & $1,11 \pm 1,00$ & $2,00 \pm 1,90$ & $2,84 \pm 2,66$ \\
\hline 31D & $4,46 \pm 1,96^{*}$ & $1,05 \pm 0,74$ & $4,59 \pm 2,55$ & $1,45 \pm 0,58$ & $2,02 \pm 2,00$ & $2,69 \pm 2,99$ \\
\hline $41 \mathrm{M}$ & $3,07 \pm 1,19^{*}$ & $1,20 \pm 0,70$ & $2,86 \pm 1,57$ & $1,00 \pm 0,85$ & $2,05 \pm 1,99$ & $2,31 \pm 2,31$ \\
\hline 41D & $3,59 \pm 1,45^{\star}$ & $1,41 \pm 0,66$ & $3,11 \pm 1,48$ & $1,01 \pm 0,99$ & $2,53 \pm 2,00$ & $2,53 \pm 2,30$ \\
\hline $42 \mathrm{M}$ & $4,40 \pm 1,84^{*}$ & $1,25 \pm 0,82$ & $4,03 \pm 2,36$ & $1,17 \pm 0,71$ & $2,64 \pm 1,95$ & $2,59 \pm 1,93$ \\
\hline $42 \mathrm{D}$ & $4,66 \pm 2,13^{*}$ & $1,22 \pm 0,92$ & $4,12 \pm 2,18$ & $1,48 \pm 0,75$ & $2,53 \pm 1,46$ & $3,01 \pm 1,49$ \\
\hline $43 \mathrm{M}$ & $3,79 \pm 2,10$ & $1,46 \pm 0,75$ & $3,64 \pm 2,71$ & $1,72 \pm 0,53$ & $2,71 \pm 1,44$ & $2,00 \pm 1,88$ \\
\hline $43 D$ & $3,06 \pm 1,61$ & $1,27 \pm 1,71$ & $2,01 \pm 1,60$ & $1,09 \pm 1,09$ & $1,84 \pm 1,28$ & $2,26 \pm 1,23$ \\
\hline Mandíbula & $3,78 \pm 1,72^{*}$ & $1,22 \pm 0,84$ & $3,53 \pm 1,73$ & $1,35 \pm 0,88$ & $2,09 \pm 1,70$ & $2,26 \pm 1,48$ \\
\hline
\end{tabular}

$\mathrm{M}=$ mesial, $\mathrm{D}=$ distal, $\mathrm{DE}=$ desviación estándar. ${ }^{*}=$ Denota diferencia estadísticamente significativa al compararse con el mismo tiempo en el grupo control $(\mathrm{P} \leq 0,05)$. $\mathrm{T} 1=30$ días, $\mathrm{T} 2=90$ días, $\mathrm{T} 3=210$ días.

ción no realizar cortes en la cresta ósea interproximal respetando $2 \mathrm{~mm}$ de esta. Se realizaron cortes en las zonas apicales en un ángulo de 45 grados. Se lavó con abundante suero fisiológico y se procedió a posicionar los colgajos y a suturar con polipropileno (6.0) para retirar 7 días después.

El control del dolor e inflamación se realizó con ibuprofeno tabletas de $400 \mathrm{mg}$ cada 8 horas por 3 días y luego con acetaminofén tabletas de $500 \mathrm{mg}$ cada 8 horas por 3 días más. Adicionalmente, se realizó terapia antibiótica con amoxicilina cápsulas de $500 \mathrm{mg}$ cada 8 horas por 7 días. Inmediatamente después de la cirugía se realizó la activación de la aparatología ortodóncica con arcos $\mathrm{Ni} \mathrm{Ti} \mathrm{0,014.}$

Análisis Estadístico. Los datos demográficos son presentados con el promedio \pm DE y la frecuencia de hombres y mujeres. Los parámetros clínicos periodontales son presentados como el promedio $\pm \mathrm{DE}$ en cada uno de los tiempos de examinación. La variable de desenlace primaria (primary outcome) fue la velocidad del mo- vimiento ortodóncico en cada uno de los grupos examinados. Las variables secundarias (secondary outcome) incluyó los cambios periodontales y en la tabla ósea cortical después de las intervenciones. Se hizo un análisis exploratorio para determinar diferencias en los parámetros clínicos periodontales y tabla ósea bucal entre grupos, se utilizaron las pruebas t-Student para muestras pareada y no pareada dependiendo del caso.

La información recolectada fue analizada con el programa estadístico SPSS versión 19 (SPSS, Inc., Chicago, IL) y se asumieron diferencias estadísticamente significativas cuando $\mathrm{P}<0,05$ ).

\section{RESULTADOS}

Un total de 10 pacientes participaron en el estudio con un promedio de edad de 25,1 años y conformados por 6 hombres y 4 mujeres. Los pacientes fueron divididos en dos grupos de 5 ( 3 hombres / 2 muje- 
ARANGO, J. D.; ROLDAN, C. M.; BURGOS, L. M.; GIRALDO, C.; GUTIÉRREZ, C. E.; SÁNCHEZ, L. A.; VILLEGAS, M.; ARANGO, D.; RESTREPO, M. \& BOTERO, J. E. Comparación clínica entre el tratamiento ortodóncico facilitado por corticotomía y ortodoncia convencional (estudio piloto). Int. J. Odontostomat., 9(2):239-248, 2015.

res) para recibir tratamiento ortodóncico + corticotomía (EXP) y tratamiento ortodóncico solo (CONT).

Los movimientos dentarios en ambos grupos fueron medidos como cambios lineales y angulares. La Tabla I muestra que el desplazamiento de los dientes,tanto superiores como inferiores, fue más rápido en el grupo EXP en comparación con el grupo CONT y esta diferencia fue estadísticamente significativa a los treinta días de seguimiento $(p<0,05)$. En algunos casos, la magnitud del movimiento en milímetros fue más del doble de lo obtenido en el grupo control. No obstante, a los 90 y 210 días de observación, no hubo diferencias estadísticamente significativas entre ambos grupos. Por otra parte, después de analizar el movimiento angular de los dientes, el cambio de posición dental medido en grados $\left(^{\circ}\right)$ fue mayor en el grupo experimental a los 30 días de observación (Tabla II). Después de 90 días no hubo diferencias entre los grupos.

El índice de irregularidad de Little (Tabla III) fue usado como herramienta de clasificación del apiñamiento, determinando la irregularidad de los diez pacientes como moderada y severa para ambos arcos. Los resultados mostraron cambios mayores entre T1 y T2 que entre T2 y T3 pero sin significancia estadística $P>0,05$.
Los parámetros clínicos periodontales pueden ser observados en la Tabla IV. En general la PD en el grupo EXP mostró una ligera disminución y se mantuvo estable después de la cirugía. En comparación, la PD en el grupo CONT aumentó $0,3 \mathrm{~mm}$ después de comenzado el tratamiento ortodóncico. Por otra parte el CAL aumentó ligeramente en ambos grupos y se mantuvo estable a largo del estudio. La cantidad de encía queratinizada (EQ) disminuyó $0,2 \mathrm{~mm}$ en promedio en ambos grupos. Además, el BOP aumentó inmediatamente después de la cirugía y disminuyó con el tiempo en el grupo EXP mientras que en el grupo CONT, el BOP aumentó con el tiempo. Por último, el IP mostró una tendencia a disminuir en el grupo EXP en contraste con el grupo CONT donde aumentó durante el tratamiento ortodóncico. No obstante, no hubo diferencias significativas en los parámetros clínicos antes y después del tratamiento entre los dos grupos (Fig. 1).

El volumen de la tabla ósea bucal medida a tres niveles de la cresta ósea antes y 7 meses después del tratamiento es presentado en la Tabla V. En general la tabla ósea bucal disminuyó $0,1 \mathrm{~mm}$ en el grupo EXP mientras que se mantuvo estable en grupo CONT. No obstante las diferencias no fueron estadísticamente significativas entre grupos (Fig. 1).

Tabla II. Movimiento angular en grados $\left(^{\circ}\right)$ por diente en el grupo corticotomía y control a los 210 días de observación.

\begin{tabular}{lcccccc}
\hline \multirow{2}{*}{ Diente } & \multicolumn{2}{c}{ Grupo corticotomía (Promedio \pm DE) } & \multicolumn{2}{c}{ Grupo control (Promedio \pm DE) } \\
\cline { 2 - 7 } & T1 & T2 & T3 & T1 & T2 & T3 \\
\hline 13 & $2,96 \pm 1,51^{*}$ & $2,06 \pm 1,91$ & $2,25 \pm 0,71$ & $0,58 \pm 0,28$ & $1,81 \pm 1,06$ & $1,55 \pm 0,52$ \\
12 & $2,52 \pm 1,60^{*}$ & $0,84 \pm 0,77$ & $2,59 \pm 1,47$ & $0,68 \pm 0,7$ & $0,89 \pm 0,52$ & $1,52 \pm 0,66$ \\
11 & $2,17 \pm 1,17$ & $0,59 \pm 0,23$ & $2,38 \pm 1,67$ & $1,65 \pm 0,43$ & $1,14 \pm 0,67$ & $1,92 \pm 1,49$ \\
21 & $2,09 \pm 1,97$ & $1,34 \pm 0,99$ & $2,17 \pm 1,37$ & $1,13 \pm, 157$ & $1,1 \pm 0,9$ & $2,22 \pm 1,64$ \\
22 & $2,43 \pm 1,48^{*}$ & $1,06 \pm 0,7^{*}$ & $1,89 \pm 1,75$ & $1,57 \pm 0,75$ & $0,28 \pm 0,25$ & $1,56 \pm 0,73$ \\
23 & $3,10 \pm 2,03$ & $1,1 \pm 1,32$ & $2,65 \pm 2,43$ & $2,23 \pm 1,08$ & $1,01 \pm 0,82$ & $1,53 \pm 0,91$ \\
33 & $3,35 \pm 2,28$ & $1,03 \pm 0,66$ & $3,28 \pm 1,37$ & $1,37 \pm 1,23$ & $1,5 \pm 1,01$ & $1,88 \pm 1,29$ \\
32 & $2,19 \pm 1,65$ & $1,33 \pm 0,75$ & $2,27 \pm 2,22$ & $1,05 \pm 0,68$ & $1,45 \pm 1,22$ & $1,72 \pm 1,26$ \\
31 & $1,97 \pm 1,69$ & $1,72 \pm 2,12$ & $2,47 \pm 1,53$ & $0,510 \pm 0,43$ & $1,27 \pm 1,24$ & $1,55 \pm 1,83$ \\
41 & $1,81 \pm 1,67$ & $1,22 \pm 1,79$ & $1,19 \pm 0,71$ & $1,14 \pm 0,71$ & $1,36 \pm 1,66$ & $2,17 \pm 1,52$ \\
42 & $3,27 \pm 0,89^{*}$ & $1,46 \pm 0,52$ & $2,35 \pm 1,52$ & $1,64 \pm 0,76$ & $1,48 \pm 1,26$ & $2,57 \pm 1,2$ \\
43 & $3,11 \pm 1,69^{*}$ & $0,71 \pm 0,72$ & $3,81 \pm 1,59$ & $1,26 \pm 0,61$ & $2,06 \pm 1,3$ & $2,04 \pm 2,2$ \\
\hline
\end{tabular}

$\mathrm{DE}=$ desviación estándar. * = Denota diferencia estadísticamente significativa al compararse con el mismo tiempo en el grupo control $(\mathrm{P} \leq 0,05)$. $\mathrm{T} 1=30$ días, $\mathrm{T} 2=90$ días, $\mathrm{T} 3=210$ días.

Tabla III. Índice de irregularidad de Little por arco en el grupo corticotomía y control a los 210 días de observación.

\begin{tabular}{lcccccc}
\hline \multirow{2}{*}{ Arco } & \multicolumn{2}{c}{ Grupo corticotomía (Promedio \pm DE) } & \multicolumn{3}{c}{ Grupo control (Promedio \pm DE) } \\
\cline { 2 - 7 } & T1 & T2 & T3 & T1 & T2 & T3 \\
\hline Maxilar & $7,74 \pm 3,52$ & $1,74 \pm 2,17$ & $9,48 \pm 3,8$ & $7,06 \pm 3,63$ & $1,22 \pm 0,65$ & $8,28 \pm 4,07$ \\
Mandibular & $9,22 \pm 2,93$ & $0,76 \pm 0,23$ & $9,98 \pm 3,09$ & $7,98 \pm 3,89$ & $1,44 \pm 1,18$ & $9,1 \pm 3,15$ \\
\hline
\end{tabular}

$\mathrm{DE}=$ desviación estándar. $\mathrm{T} 1=30$ días, $\mathrm{T} 2=90$ días, $\mathrm{T} 3=210$ días. 
ARANGO, J. D.; ROLDAN, C. M.; BURGOS, L. M.; GIRALDO, C.; GUTIÉRREZ, C. E.; SÁNCHEZ, L. A.; VILLEGAS, M.; ARANGO, D.; RESTREPO, M. \& BOTERO, J. E.

Comparación clínica entre el tratamiento ortodóncico facilitado por corticotomía y ortodoncia convencional (estudio piloto). Int. J. Odontostomat., 9(2):239-248, 2015.

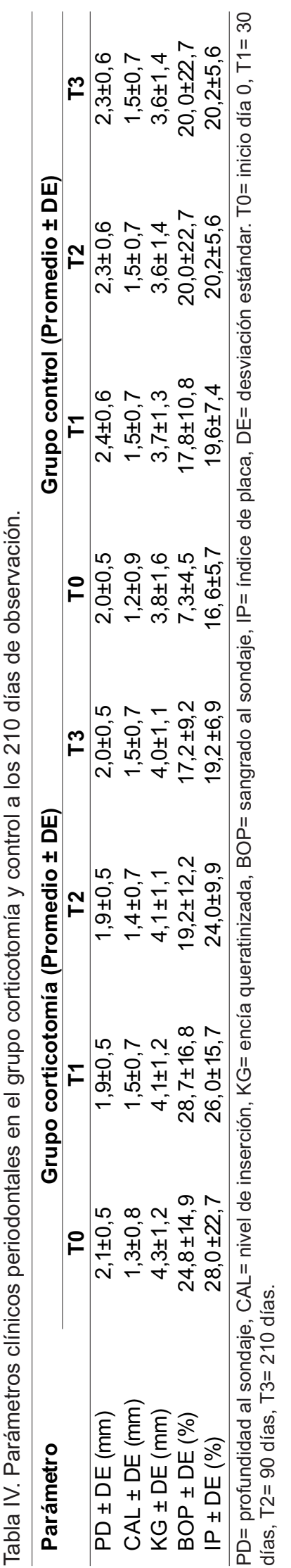

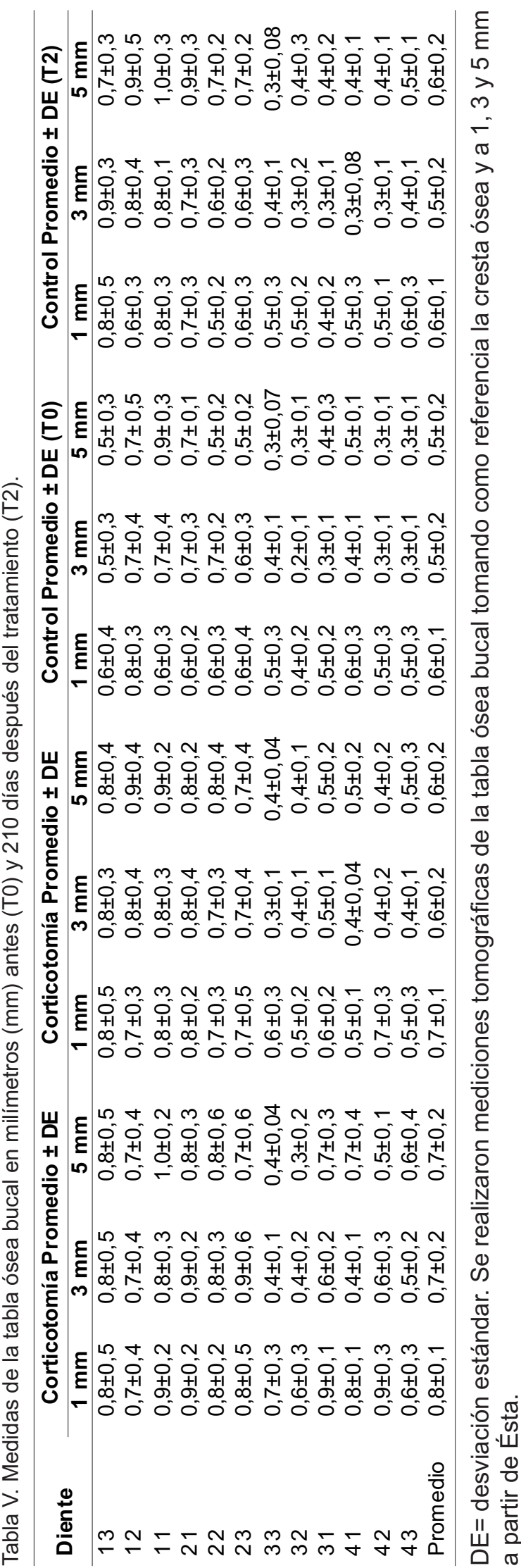

\section{DISCUSION}

El tratamiento ortodóncico generalmente tiene una duración de 2 años, pero este puede variar dependiendo de la severidad de la maloclusión. Teniendo esto en cuenta, se ha venido estudiando y desarrollando la corticotomía como procedimiento coadyuvante para acelerar el movimiento ortodóncico y por ende disminuir el tiempo de tratamiento. Estudios experimentales en animales (Wang et al.; Mostafa et al., 2009) reportan como consecuencia de la corticotomía un aumento en la velocidad de movimiento dental, con tasas de movimiento promedio en el grupo de corticotomía de $4,67 \pm 0,58 \mathrm{~mm}$ vs. $2,33 \pm 0,58$ $\mathrm{mm}$ en el grupo control. No obstante, su seguridad y aplicación en humanos todavía esta en estudio. Algunos reportes de casos en humanos con corticotomía (Wilcko et al., 2009; Chung et al.; Akay et al.), evidencian que el tiempo de tratamiento oscila entre los 4 y 18 meses según la complejidad de la maloclusión. Lo anterior sugiere que la corticotomía puede ser una alternativa viable en algunos pacientes.

\section{EI}

protocolo ortodóncico para la alineación y nivelación propuesto para el presente estudio tuvo en consideración los niveles de aplicación de fuerzas. Los resultados de las investigaciones de ortodoncia acelerada muestran variaciones en la intensidad de la fuerza desde los 60 a los $900 \mathrm{~g}$. 


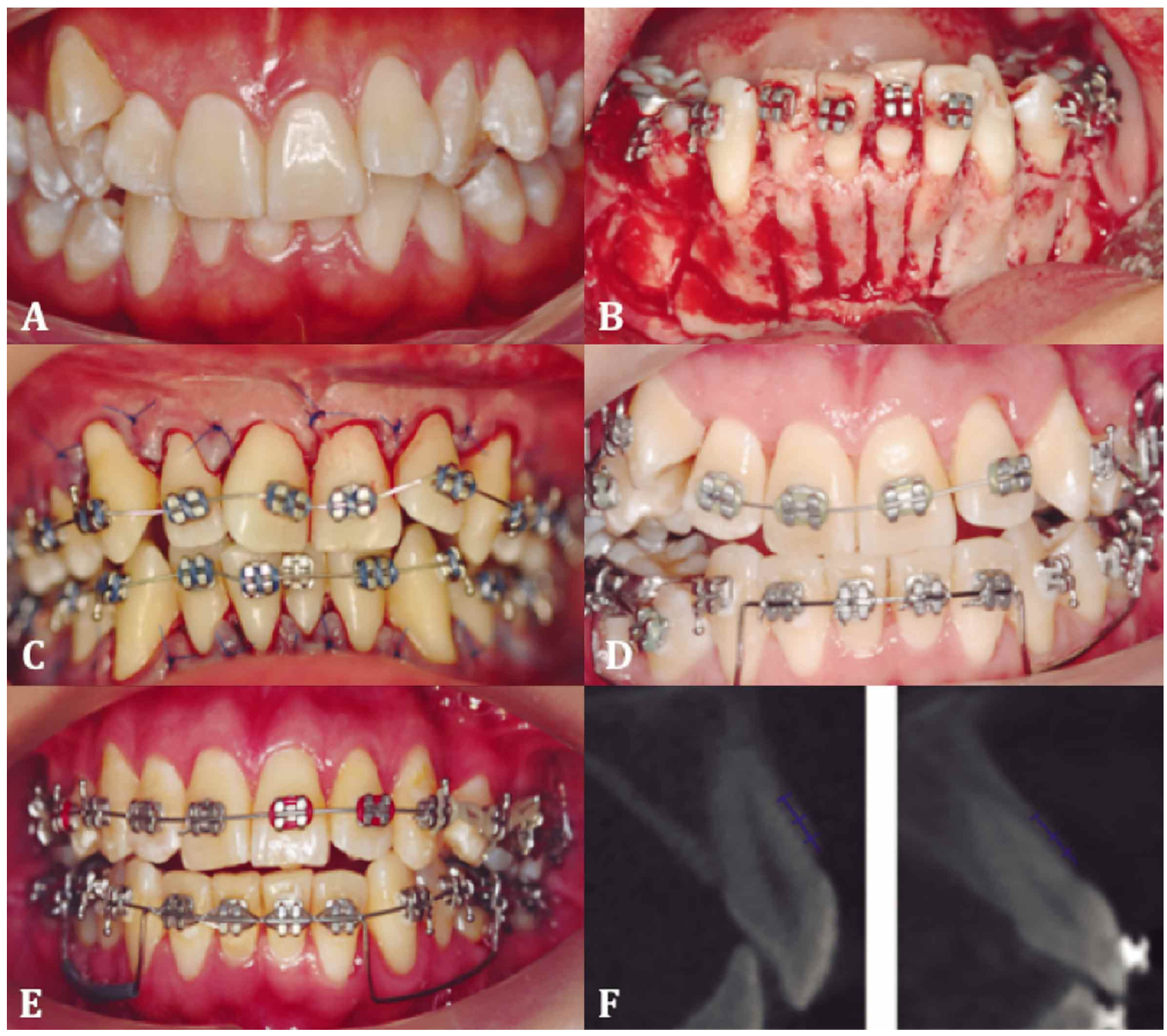

Fig. 1. Paciente grupo corticotomía. A) Vista preoperatoria: se aplico solución de Schiller para determinar el ancho de la encía queratinizada y parámetros periodontales. B) Vista quirúrgica: se observa colgajo a espesor total en la zona anteroinferior y las corticotomías realizadas siguiendo los ejes longitudinales de los dientes a $3 \mathrm{~mm}$ de la cresta ósea. C) Suturas en posición: se observan suturas no reabsorbibles 5.0 y la activación de la ortodoncia con la colocación del arco de Ni Ti 0.014 . D) Vista postquirúrgica dos meses depués de la cirugía. e) Vista postquirúrgica 7 meses despues de la cirugia. F) Vista radiografica: se observa la tabla osea bucal antes de la cirugia y 7 meses después.

(Fisher, 2007; Akay et al.) sin embargo, la magnitud de fuerza óptima para aplicar después de la corticotomía aún no está clara. Los niveles de fuerza aplicados en ambos grupos, se mantuvieron en un rango fisiológico entre los 15 y $25 \mathrm{~g}$ por diente, considerando el protocolo ejecutado en relación con la secuencialidad de los arcos de Níquel Titanio y la inclusión en el arco según la solución gradual del apiñamiento.
Como se ha expuesto previamente, este estudio obtuvo un valor promedio de movimiento lineal durante el primer mes para el grupo experimental en el maxilar de $3,56 \pm 1,90 \mathrm{~mm}$ vs. $1,20 \pm 0,85 \mathrm{~mm}$ en el grupo control, y en la mandíbula para el grupo experimental de $3,78 \pm 1,72 \mathrm{~mm}$ vs. $1,35 \pm 0,88 \mathrm{~mm}$ para el grupo control. Se evidencia una mayor magnitud de movimiento para el grupo experimental, en este intervalo de medición. Adicionalmente, al analizar los resultados lineales por 
ARANGO, J. D.; ROLDAN, C. M.; BURGOS, L. M.; GIRALDO, C.; GUTIÉRREZ, C. E.; SÁNCHEZ, L. A.; VILLEGAS, M.; ARANGO, D.; RESTREPO, M. \& BOTERO, J. E.

Comparación clínica entre el tratamiento ortodóncico facilitado por corticotomía y ortodoncia convencional (estudio piloto). Int. J. Odontostomat., 9(2):239-248, 2015.

cada diente se evidencia como el mayor promedio de movimiento se dio en los incisivos tanto maxilares como mandibulares, mientras que los caninos no mostraron diferencias estadísticamente significativas. Cuando se analizan los valores arrojados por las mediciones angulares a los 90 días de seguimiento se evidencian valores más altos en grados para el grupo experimental que para el grupo control, tanto para el maxilar como para la mandíbula, aunque las diferencias en general no fueron estadísticamente significativas. Es importante la correlación de los resultados obtenidos con el principio biológico FAR que ocurre en los primeros 3 meses después de la corticotomía. Estos resultados apoyan los hallazgos del estudio de Aboul-Ela et al. (2011) donde se expone que la distancia acumulativa de movimiento fue significativamente mayor en el grupo con corticotomía, así, en el primer mes fue de 1,89 vs. 0,75 $\mathrm{mm}$, a los dos meses de 1,83 vs 0,86 , a los tres meses de 1,07 vs $0,93 \mathrm{~mm}$ y en el cuarto mes de 0,89 vs. 0,85 $(P=0,01)$. Al analizar estos valores como una sumatoria equivaldría a una distancia acumulativa de movimiento en los cuatro primeros meses, periodo FAR, de $5,68 \mathrm{~mm}$ para el grupo con corticotomía vs $2,71 \mathrm{~mm}$ para el grupo control respectivamente. Por su parte, Fischer reportó una tasa de movimiento que fue significativamente más alta en el grupo con corticotomía 0,02656-0,036mm vs. sin corticotomía $0,185-0,014 \mathrm{~mm}$ por semana $(\mathrm{P}=$ $0,001)$. Estos autores coinciden con los resultados arrojados por el presente estudio ya que definen la tendencia a un mayor movimiento en las 12 semanas siguientes al procedimiento quirúrgico, así, los resultados obtenidos durante la fase de alineación y nivelación apoyan el concepto que la corticotomía modifica la biología del movimiento dentario basados en estudios inmunohistoquímicos que soportan una respuesta de remodelado óseo acelerado posterior al estímulo (Wang et al., 2009).

No obstante, la corticotomía en si consiste en un procedimiento quirúrgico importante que puede tener impacto sobre los tejidos periodontales. Este estudio clíni$\mathrm{co}$, en su protocolo incluye la osteotomía de solamente la cortical bucal, no recurrió al uso de injertos y evaluó los cambios en los parámetros clínicos periodontales. La medición de los parámetros clínicos periodontales (PD, CAL, BOP, IP) son la forma actualmente empleada para monitorear la salud y estabilidad del periodonto. $\mathrm{Si}$ bien, no hubo diferencias en los parámetros clínicos periodontales entre grupos, fue posible observar algunos cambios menores que son propios de los procedimientos quirúrgicos periodontales. Por una parte la PD disminuyó en el tiempo en el grupo experimental mientras que el CAL permaneció estable. Por otra parte, la cantidad de KG disminuyó ligeramente, asociado posiblemente al procedimiento quirúrgico y el movimiento de inclinación bucal de los dientes. No obstante, al comparar esto hallazgos con el grupo control, no se observaron diferencias estadística y clínicamente significativas. Estos resultados sugieren una buena estabilidad de los tejidos periodontales cuando se encuentran sanos y se asocia con una buena seguridad del procedimiento terapéutico. Es evidente que factores como la habilidad del clínico, las condiciones en las cuales se realiza el procedimiento quirúrgico, la forma como cicatriza el tejido y la severidad del apiñamiento son altamente influyentes en la seguridad y estabilidad de la corticotomía. En el presente estudio se incluyeron pacientes con apiñamiento anterior moderado a severo, pero sería necesario realizar más estudios en casos severos para determinar los alcances de la técnica. Hasta nuestro conocimiento este es el único estudio clínico que ha evaluado los cambios periodontales en pacientes con ortodoncia facilitado por corticotomía adicional al estudio de Nowzari et al.

Una de las limitantes de la corticotomía es el volumen de la tabla ósea bucal. Dado que el procedimiento requiere del levantamiento del colgajo a espesor total más allá de los ápices dentarios, estaría contraindicado en zonas con ausencia de las tablas óseas bucales lo cual resultaría en recesión del tejido marginal. En el presente estudio se incluyeron pacientes con periodonto medio-grueso que sugiriera la presencia de una tabla ósea bucal mínima. Esto fue confirmado en las tomografías y durante la cirugía. Se encontró que no hubo cambios clínicamente significativos en el volumen óseo bucal, que varió entre 1,0-0,3 mm en la zona más coronal, 210 días después del procedimiento quirúrgico. Así mismo, no hubo diferencias con el grupo control. Esto indica que el procedimiento puede realizarse de manera segura en zonas donde hay una cantidad mínima de hueso alveolar bucal. No obstante, algunos autores recomiendan el uso de material de injerto óseo sobre la zona operada (Nowzari et al.). Desafortunadamente la falta de control en dichos estudios aporta poca evidencia al uso indicado de materiales de injerto óseo y sigue más bien siendo una cuestión de elección del clínico. Esto demuestra que se necesitan más estudios con el uso y sin uso de materiales de injerto. En nuestro estudio, en ningún caso hubo efectos negativos a los tejidos periodontales o al hueso alveolar. Adicionalmente, es necesario más estudios con mayor cantidad de pacientes ya que este estudio tienen la limitante de la poca cantidad de pacientes. Esto último debido a la naturaleza quirúrgica que no permitió hacer una aleatorización de los pacientes. 
ARANGO, J. D.; ROLDAN, C. M.; BURGOS, L. M.; GIRALDO, C.; GUTIÉRREZ, C. E.; SÁNCHEZ, L. A.; VILLEGAS, M.; ARANGO, D.; RESTREPO, M. \& BOTERO, J. E. Comparación clínica entre el tratamiento ortodóncico facilitado por corticotomía y ortodoncia convencional (estudio piloto). Int. J. Odontostomat., 9(2):239-248, 2015.

\section{CONCLUSIONES}

La corticotomía acelera el movimiento ortodóncico durante el primer mes postquirúrgico con lo cual se puede reducir el tiempo de tratamiento en pacientes con apiñamiento severo. Adicionalmente, los parámetros clínicos periodontales y el volumen de la tabla ósea bucal de los dientes sometidos a corticotomía se mantienen estables después del procedimiento. No obstante, no es recomendable en biotipos periodontales delgados.

\section{AGRADECIMIENTOS.}

Este estudio fue financiado por la Universidad de Antioquia. Los autores no reportan conflictos de interés.

ARANGO, J. D.; ROLDAN, C. M.; BURGOS, L. M.; GIRALDO, C.; GUTIÉRREZ, C. E.; SÁNCHEZ, L. A.; VILLEGAS, M.; ARANGO, D.; RESTREO, M. \& BOTERO, J. E. Clinical comparison of orthodontic treatment facilitated by corticotomy and conventional orthodontics (pilot study). Int. J. Odontostomat., 9(2):239-248, 2015.

Abstract: The aim of this study was to compare clinically speed orthodontic movement and changes in periodontal parameters in patients with conventional orthodontics and orthodontics with corticotomy for the treatment of anterior dental crowding. A total of 10 patients participated in the study: 5 patients in the orthodontics and corticotomy group (EXP) and 5 in the orthodontics patient group (CONT). Complete clinical examination included radiographic, periodontal and study models analysis was carried out. Measurements on periodontal changes, buccal bone plate, linear and angular movement were measured at baseline at 30,90 and 210 days after the procedures. Differences between groups were established with the tStudent for paired and unpaired samples depending on the case. Teeth undergoing orthodontic corticotomy showed greater speed of movement compared to the control group during the first 30 days examination $(P<0.05)$. There were no statistically significant differences in periodontal clinical parameters and thickness of the buccal bone plate between the groups. In conclusion, accelerated corticotomy orthodontic movement during the first 30 days postoperative, can reduce the treatment period in patients with severe crowding. Additionally, clinical periodontal parameters and volume of the buccal bone plate teeth undergoing corticotomy remained stable after the procedure.

KEY WORDS: corticotomy, accelerated orthodontics, clinical periodontal parameters, regional acceleratory phenomenon (RAP).

\section{REFERENCIAS BIBLIOGRÁFICAS}

Aboul-Ela, S. M.; El-Beialy, A. R.; El-Sayed, K. M.; Selim, E. M.; El-Mangoury. N. H. \& Mostafa, Y. A. Miniscrew implant-supported maxillary canine retraction with and without corticotomy-facilitated orthodontics. Am. J. Orthod. Dentofacial Orthop., 139(2):252-9, 2011.

Akay, M. C.; Aras, A.; Günbay, T.; Akyalçin, S. \& Koyuncue, B. O. Enhanced effect of combined treatment with corticotomy and skeletal anchorage in open bite correction. J. Oral. Maxillofac. Surg., 67(3):563-9, 2009.

Buschang, P. H.; Campbell, P. M. \& Ruso, S. Accelerating tooth movement with corticotomies: is it possible and desirable? Semin. Orthod., 18(4):286-94, 2012.

Buttke, T. M. \& Proffit, W. R. Referring adult patients for orthodontic treatment. J. Am. Dent. Assoc., 130(1):739, 1999.

Byloff, F. K. \& Mossaz, C. F. Skeletal and dental changes following surgically assisted rapid palatal expansion. Eur. J. Orthod., 26(4):403-9, 2004.

Christensen, L. \& Luther, F. Adults seeking orthodontic treatment: expectations, periodontal and TMD issues. Br. Dent. J., 218(3):111-7, 2015.

Chung, K. R.; Kim, S. H. \& Lee, B. S. Speedy surgicalorthodontic treatment with temporary anchorage devices as an alternative to orthognathic surgery. Am. J. Orthod. Dentofacial Orthop., 135(6):787-98, 2009.

Consolaro, A. In adults: $47.2 \%$ have periodontitis! How about in orthodontic patients? Dental Press J. Orthod., 18(1):3-5, 2013

Dhar, V.; Jain, A.; Van Dyke, .T. E. \& Kohli, A. Prevalence of gingival diseases, malocclusion and fluorosis in school-going children of rural areas in Udaipur district. J. Indian Soc. Pedod. Prev. Dent., 25(2):103-5, 2007.

Fischer, T. J. Orthodontic treatment acceleration with corticotomy-assisted exposure of palatally impacted canines. Angle Orthod., 77(3):417-20, 2007.

Gantes, B.; Rathbun, E. \& Anholm, M. Effects on the periodontium following corticotomy-facilitated orthodontics. Case reports. J. Periodontol., 61(4):2348,1990

Hanna, A.; Chaaya, M.; Moukarzel, C.; El Asmar, K.; Jaffa, M. \& Ghafari, J. G. Malocclusion in elementary school children in beirut: severity and related social/behavioral factors. Int. J. Dent., 2015:351231, 2015. 
ARANGO, J. D.; ROLDAN, C. M.; BURGOS, L. M.; GIRALDO, C.; GUTIÉRREZ, C. E.; SÁNCHEZ, L. A.; VILLEGAS, M.; ARANGO, D.; RESTREPO, M. \& BOTERO, J. E.

Comparación clínica entre el tratamiento ortodóncico facilitado por corticotomía y ortodoncia convencional (estudio piloto). Int. J. Odontostomat., 9(2):239-248, 2015.

Januário, A. L.; Duarte, W. R.; Barriviera, M.; Mesti, J. C.; Araújo, M. G. \& Lindhe, J. Dimension of the facial bone wall in the anterior maxilla: a cone-beam computed tomography study. Clin. Oral Implants Res., 22(10):1168-71, 2011.

Jung, M. H. An evaluation of self-esteem and quality of life in orthodontic patients: Effects of crowding and protrusion. Angle Orthod., 85(5):812-9, 2015.

Lee, J. K.; Chung, K. R. \& Baek, S. H. Treatment outcomes of orthodontic treatment, corticotomyassisted orthodontic treatment, and anterior segmental osteotomy for bimaxillary dentoalveolar protrusion. Plast. Reconstr. Surg., 120(4):1027-36, 2007.

Little, R. M. The irregularity index: a quantitative score of mandibular anterior alignment. Am. J. Orthod., 68(5):554-63, 1975.

Mostafa, Y. A.; Mohamed Salah Fayed, M.; Mehanni, S.; ElBokle, N. N. \& Heider, A. M. Comparison of corticotomy-facilitated vs standard tooth-movement techniques in dogs with miniscrews as anchor units. Am. J. Orthod. Dentofacial Orthop., 136(4):570-7, 2009.

Nowzari, H.; Yorita, F. K. \& Chang, H. C. Periodontally accelerated osteogenic orthodontics combined with autogenous bone grafting. Compend. Contin. Educ. Dent., 29(4):200-6, 2008.

Owen, A. H. 3rd. Accelerated Invisalign treatment. J. Clin. Orthod., 35(6):381-5, 2001.

Panwar, M.; Jayan, B.; Arora, V. \& Singh, S. Orthodontic management of dentition in patients with periodontally compromised dentition. J. Indian Soc. Periodontol., 18(2):200-4, 2014.

Siluvai, S.; Kshetrimayum, N.; Reddy, C. V.; Siddanna, S.; Manjunath, M. \& Rudraswamy, S. Malocclusion and related quality of life among 13- to 19-year-old students in Mysore City - a cross-sectional study. Oral Health Prev. Dent., 13(2):135-41, 2015.

Wang, L.; Lee, W.; Lei, D. L.; Liu, Y. P.; Yamashita, D. D. \& Yen, S. L. Tisssue responses in corticotomyand osteotomy-assisted tooth movements in rats: histology and immunostaining. Am. J. Orthod. Dentofacial Orthop., 136(6):770.e1-11, 2009.
Wilcko, M. T.; Wilcko, W. M.; Pulver, J. J.; Bissada, N. F. \& Bouquot, J. E. Accelerated osteogenic orthodontics technique: a 1-stage surgically facilitated rapid orthodontic technique with alveolar augmentation. J. Oral Maxillofac. Surg., 67(10):2149-59, 2009.

Wilcko, W. M.; Wilcko, T.; Bouquot, J. E. \& Ferguson, D. J. Rapid orthodontics with alveolar reshaping: two case reports of decrowding. Int. J. Periodontics. Restorative Dent., 21(1):9-19, 2001.

Dirección para Correspondencia:

Juan David Arango

Facultad de Odontología Universidad de Antioquia

Calle 70 No. 52-21

Medellín

COLOMBIA

Email: juandavid822@hotmail.com

Recibido : 16-03-2015

Aceptado: 10-07-2015 